\title{
Metabolic Abnormalities Associated with the Use of Protease Inhibitors and Non-nucleoside Reverse Transcriptase Inhibitors
}

\author{
${ }^{1,2}$ Madhu N. Rao, ${ }^{1,2}$ Grace A. Lee and ${ }^{1,2}$ Carl Grunfeld \\ ${ }^{1}$ Department of Medicine, University of California at San Francisco \\ ${ }^{2}$ Metabolism and Endocrine Sections, Department of Veteran Affairs Medical Center, San Francisco
}

\begin{abstract}
The use of protease inhibitors and non-nucleoside reverse transcriptase inhibitors for the treatment of HIV infection and AIDS has been associated with multiple abnormalities in glucose and lipid metabolism. Specifically, these abnormalities include insulin resistance, increased triglycerides and increased LDL cholesterol levels. The metabolic disturbances are due to a combination of factors, including the direct effect of medications, restoration to health and HIV disease, as well as individual genetic predisposition. Of the available anti-retroviral medications, indinavir has been associated with causing the most insulin resistance and ritonavir with causing the most hypertriglyceridemia.
\end{abstract}

Key words: HIV, glucose, lipids, protease inhibitor, antiretroviral

\section{INTRODUCTION}

The use of highly active antiretroviral therapy (HAART) has significantly reduced morbidity and mortality in HIV infection and AIDS ${ }^{[1]}$. But the use of antiretroviral medications (ARVs), specifically protease inhibitors (PIs), has been associated with various metabolic and morphological abnormalities. Abnormalities in glucose metabolism, alterations in lipids and changes in body fat deposition (known as lipodystrophy) have been reported starting in $1997^{[2-5]}$. Although some patients on ARVs may have multiple metabolic and morphological abnormalities, it is clear that many show only one aspect.

In this review, we will discuss the alterations in glucose and lipid metabolism caused by PIs and nonnucleoside reverse transcriptase inhibitors (NNRTIs). Although the changes in fat distribution that occur in association with AIDS and ARV treatment may impact metabolism, they should be viewed as distinct entities and are therefore not within the scope of this review. Furthermore, it is important to separate and analyze independently the metabolic abnormalities due to HIV infection itself, restitution of health and medications. We will first review the metabolic changes that occur as a result of HIV infection alone, followed by the specific effects of ARVs on glucose and lipid metabolism.

Metabolic abnormalities in HIV infection: HIV infection itself has been shown to cause changes in glucose and lipid metabolism. Before the era of effective antiretroviral therapy, Hommes et al. demonstrated that patients with HIV infection had increased insulin sensitivity compared to healthy, HIV negative controls using the gold standard of the euglycemic, hyperinsulinemic clamp ${ }^{[6]}$. The increased insulin sensitivity is likely secondary to an increase in non-oxidative glucose disposal in HIV infection ${ }^{[7,8]}$. Although some studies suggest a decrease in insulin sensitivity rather than an increase, it is important to note that that these were done using other less accurate methods (fasting glucose levels, fasting insulin levels, OGTT) rather than the gold standard of the clamp.

Lipid metabolism is, similarly, altered in the presence of HIV infection and AIDS. The earliest change in lipid metabolism is decreased HDL cholesterol (HDL-c) and apo A-1 ${ }^{[9-12]}$. With disease progression, LDL cholesterol (LDL-c) levels decrease, followed by an increase in triglyceride (TG) and VLDL-c levels with development of $\operatorname{AIDS}^{[9]}$. The magnitude of the LDL-c change is less than that of HDL- $\mathrm{c}^{[9,10,12]}$, thus promoting an atherogenic lipid profile. Triglyceride levels in AIDS are significantly increased $(33-153 \%)^{[9,12,13]}$ due to a combination of both decreased TG clearance ${ }^{[9]}$ and increased VLDL production $^{[14]}$. The increased TG level has been shown to correlate with elevated interferon- $\alpha$ levels ${ }^{[9,12]}$ the host response to viral infection. Lastly, increased TGs have been associated with an increased prevalence of LDL-b phenotype in patients with AIDS ${ }^{[15]}$. This constellation of changes in HIV infection and AIDS (increased TG, decreased HDL-c and smaller LDL particles) are consistent with a dyslipidemic, proatherogenic profile.

Assessment of glucose metabolism: Various studies on the effects of ARVs on glucose metabolism have found differing results. Part of the discrepancy is due to different methods used to determine insulin resistance

Corresponding Author: Carl Grunfeld, University of California, San Francisco, Veterans Affairs Medical Center, Metabolism Section 111F, 4150 Clement Street, San Francisco, CA 94121, USA, Tel: 415-750-2005, Fax: 415-750-6927 
that vary with respect to sensitivity, accuracy and the specific variable being measured. Insulin resistance is a state in which there is a decreased biological effect for a given concentration of endogenous or exogenous insulin, i.e. a decrease in sensitivity to insulin. The euglycemic, hyperinsulinemic clamp is considered the gold standard method for determining insulin sensitivity. The subject is infused with exogenous insulin and plasma glucose is "clamped" at a specific, normoglycemic level by titrating a glucose infusion. The hyperglycemic clamp, based on this same concept but without the insulin infusion, is used primarily to determine pancreatic $\beta$-cell secretory function, but can also be used to measure insulin resistance. The primary disadvantage of the clamp methods is the intensive time, labor and cost.

Other methods of quantifying insulin resistance include the minimal model (typically based on the frequently sampled IV glucose tolerance test), insulin tolerance test and homeostasis model assessment (HOMA). The minimal model involves an IV glucose load and subsequent glucose and insulin measurements; its primary disadvantage is that it requires adequate endogenous insulin secretion (which is often abnormal in patients on PIs ${ }^{[16-18]}$ ) and the coefficient of variation for the minimal model is $20 \%$ within a subject ${ }^{[19]}$. The insulin tolerance test determines insulin sensitivity by assessing the rate of decline of plasma glucose after an intravenous dose of insulin; its primary disadvantage is the possibility of hypoglycemia and the risk of counterregulatory hormones (glucagon, catecholamines, cortisol) distorting the results. HOMA is a mathematical model to assess insulin resistance based on fasting serum glucose (FSG) and fasting insulin concentrations; unlike other measurements, HOMA is a determination of basal (rather than stimulated) insulin sensitivity. Although HOMA can be useful in epidemiology studies with a large number of patients, it has a higher coefficient of variation within an individual $(30 \%)^{[20]}$ and is thus less accurate in quantifying insulin resistance.

Other tests commonly used to measure glucose metabolism include the oral glucose tolerance test (OGTT), fasting serum glucose (FSG) and random serum glucose. These tests are useful in defining clinical states such as diabetes, impaired fasting glucose and impaired glucose tolerance. In a research setting, the OGTT (but not FSG or random glucose) can be modified to estimate insulin resistance by sequentially measuring insulin as well as glucose levels after the administration of a standard oral glucose load. The OGTT is the most sensitive of these tests followed by FSG and lastly, the random serum glucose.

Prevalence of abnormal glucose metabolism: Soon after the introduction of protease inhibitors, reports of abnormal glucose metabolism began to emerge. Dube et al. first reported hyperglycemia (defined as random glucose $>180 \mathrm{mg} \mathrm{dL}^{-1}$ ) in 7 out of 1050 patients $(1 \%)$ on $\mathrm{PIs}^{[2]}$ and this was quickly followed by multiple other reports of hyperglycemia and diabetes mellitus (DM) ${ }^{[5,21,22]}$. While a wide variability in the prevalence of glucose abnormalities was reported, some of the variability can be attributed to differences in methods of assessment or definitions of glucose abnormalities.

The prevalence of diabetes as defined by either random glucose $>200 \mathrm{mg} \mathrm{dL}^{-1}$ or $\mathrm{FSG}>/=126 \mathrm{mg} \mathrm{dL}^{-1}$ has ranged from $2 \%{ }^{[5,23,24]}$ to $6-7 \%^{[25-27]}$. Using a more sensitive technique, the OGTT, Carr et al. found that diabetes occurred in $7 \%$ of patients on PIs ${ }^{[25]}$. Also using the OGTT, Behrens et $a l^{[16]}$ reported the highest prevalence of DM (13\%) in patients who had been receiving PIs for a mean of 18 months. The prevalence of impaired glucose tolerance (IGT) has been reported to be $16 \%^{[25]}, 17 \%^{[27]}$ and $46 \%^{[16]}$ in various studies.

Insulin resistance: A well designed study by Walli et al. used the insulin tolerance test in 67 patients on PIs and found that $61 \%$ were insulin resistant ${ }^{[22]}$. The degree to which each individual PI causes insulin resistance varies widely. It is likely that indinavir (IDV) has the most potent effect on glucose metabolism, increasing insulin resistance by over $30 \%^{[28,29]}$ as determined by HOMA and minimal model. Although some studies conclude that IDV does not change glucose metabolism, these studies used less sensitive techniques such as random glucose and $\mathrm{FSG}^{[30,31]}$.

Because studies of insulin resistance in HIV positive patients can be skewed by factors such as immune reconstitution, return to health and changes in body composition with long term therapy, studies using HIV negative subjects can help in elucidating the direct effects of PI. Noor et al. performed euglycemic clamp studies in HIV negative patients who were given IDV. After a single dose of IDV, resistance to insulinmediated glucose disposal increased by $34 \%{ }^{[32]}$. After 4 weeks of IDV, resistance to insulin-mediated glucose disposal increased by $17 \%$, insulin levels increased by $36 \%$ and HOMA by $47 \%{ }^{[33]}$ as clearly shown in this study, the changes in HOMA, insulin-mediated glucose disposal and insulin levels are not interchangeable.

Nelfinavir (NFV) may also increase insulin resistance $^{[34]}$. Studies of the effect of amprenavir (APV) and lopinavir/ritonavir (LPV/r) on insulin resistance have been conflicting. Dube et al. showed a trend towards increased insulin resistance (using the FSIVGTT) in HIV patients treated with 48 weeks of APV, but not at earlier times ${ }^{[35]}$. Euglycemic clamp studies of LPV/r have had varying results. In HIV negative men, no change in insulin sensitivity by clamp was seen after 4 weeks of $\mathrm{LPV} / \mathrm{r}^{[36]}$, whereas a study of five days of $\mathrm{LPV} / \mathrm{r}$ showed a $24 \%$ increase in insulin resistance by clamp ${ }^{[37]}$. Studies using the less sensitive techniques of random glucose and FSG have shown no change with $\mathrm{LPV} / \mathrm{r}^{[31,38,39]}$. Lastly, work with atazanavir 
(ATZ) does not cause insulin resistance in HIV negative subjects after 5 days of treatment ${ }^{[37]}$.

How it is possible that certain PIs cause an acute blockade of insulin mediated glucose disposal in humans, but the blockade is ameliorated or lost with four weeks of treatment? Hormones and adipocytokines, such as adiponectin, may be responsible. Adiponectin is a hormone secreted by adipocytes which correlates with insulin sensitivity. Lee et al. showed that adiponectin levels were increased after four weeks of either LPV/r or IDV treatment in HIV negative men, but the increase was higher in $\mathrm{LPV} / \mathrm{r}^{[40]}$. Both PIs acutely induce insulin resistance, but after four weeks LPV/r did not cause insulin resistance, whereas IDV still did, albeit less than acutely ${ }^{[32,33,36]}$.

Mechanism of action of PI-induced inhibition of insulin mediated glucose disposal - In vitro studies: One mechanism by which PIs may induce insulin resistance is by acutely blocking the GLUT4 glucose transporter. The binding of insulin to its receptor initiates a signaling cascade that ultimately results in the translocation of GLUT4 (the primary transporter of glucose in response to insulin) from intracellular vesicles to the plasma membrane. PIs decrease insulin stimulated, but not basal, glucose uptake in cells ${ }^{[41-44]}$. This decrease in glucose uptake is rapidly reversible ${ }^{[41]}$, occurs at physiological doses of drug ${ }^{[45]}$ and occurs in Xenopus laevis oocytes expressing GLUT4 only ${ }^{[41]}$. Further evidence for the direct inhibition of GLUT4 by PIs comes from studies showing no change in any of the mediators of insulin signaling and no change in plasma membrane levels of GLUT4 $4^{[41,42]}$.

Other actions of PIs: PIs may also alter glucose metabolism by suppressing insulin secretion from pancreatic beta cells ${ }^{[16-18]}$. Using a hyperglycemic clamp, Woerle et al. showed that initial phase 1 insulin secretion is decreased by $25 \%$ in patients on $\mathrm{Pis}^{[17]}$. In vitro studies have also shown that some PIs (including IDV, APV, RTV and NFV) inhibit insulin secretion by pancreatic beta cells ${ }^{[46,47]}$.

The IDV-induced increase in fasting insulin levels and HOMA ${ }^{[33]}$ cannot be explained by the inhibition of insulin-mediated glucose disposal. Thus it is important to recognize that IDV has also been shown to increase hepatic glucose production and glycogenolysis, as well as blunting their suppression by insulin ${ }^{[48]}$.

Effect of NNRTIs on glucose metabolism: Although non-nucleoside reverse transcriptase inhibitors (NNRTIs) have not been studied as well as PIs, there are some studies of the effect on glucose metabolism of "switching" from a PI-based regimen to nevirapine (NVP) or efavirenz (EFV). The majority of studies show that NVP and EFV cause less insulin resistance than PIs; insulin resistance decreased by $28-67 \%$ (as assessed by fasting insulin resistance index or fasting glucose-insulin ratio) when HIV positive patients were switched from PIs to NNRTIS ${ }^{[49-51]}$. Studies directly assessing insulin resistance caused by NNRTIs (rather than that relative to PIs) using the gold standard of the glucose clamp have not yet been performed. Of note, switch studies in patients with lipodystrophy have had widely differing results.

Assessment of Lipid metabolism: As with glucose metabolism, the literature on lipid metabolism and HAART is widely varied. Part of this variability is due to differences in measurement techniques. For example, TG levels should be measured in the fasting state because the levels are significantly increased by dietary fat intake and may vary due to the nature and timing of the meal; thus "random" TG levels should not be used. Likewise, in patients with significant hypertriglyceridemia $\left(>400 \mathrm{mg} \mathrm{dL}^{-1}\right)$, LDL-c should be measured directly, rather than estimated from the Friedewald formula. Although directly measured LDL$c$ is ideal in a research setting, calculated LDL-c is acceptable if patients do not have hypertriglyceridemia.

In addition to cholesterol and TG levels, a few studies have analyzed the effect of PIs on various apolipoproteins (the protein moieties of lipoproteins). ApoA (I, II and IV) is predominantly associated with HDL, apoB with LDL, VLDL and chylomicrons, apoC II and III with chylomicrons and VLDL and apoE with IDL and chylomicron remnants.

Effect of PIs on Lipid metabolism: With the introduction of HAART, one might have expected a reversal of the lipid abnormalities (elevated TG, decreased LDL and HDL) observed in HIV infection and AIDS. Instead, the lipid profiles of patients on PIs have been varied and reflect not only the intrinsic effects of PIs on lipid metabolism, but also immune reconstitution and a return to health. Hyperlipidemia (as defined by elevated total cholesterol or TG) has been reported to occur in $71-78 \%$ of patients on PI-based therapy, while hypertriglyceridemia has been reported in $28-50 \%{ }^{[16,25,27,52]}$. As a group, the PIs cause increased total cholesterol, TG and LDL-c levels ${ }^{[17,29,52,53]}$, while HDL levels are mostly unchanged ${ }^{[5,29]}$. Despite the generalization, studies have shown that individual PIs have different effect on lipids.

Triglycerides: Some PIs further increase TG levels beyond what is seen in AIDS. Ritonavir has the largest effect, increasing TG levels by $101-300 \%{ }^{[30,54,55]}$. In an early study of 543 HIV patients on full dose RTV, 13\% developed fasting TG levels of $>1500 \mathrm{mg} \mathrm{dL}^{-1}{ }^{[56]}$. In studies of HIV negative patients, in whom infection and inflammation are not confounding factors, RTV has also been shown to increase TG levels by $146 \%{ }^{[55]}$; most of this increase was in VLDL particles. LPV/r also increases TG levels by $27-108 \%$ in both HIV positive 
Table 1: Effect of PIs and NNRTIs on Lipid and Glucose Metabolism in HIV-infected patients

\begin{tabular}{|c|c|c|c|c|c|}
\hline Drug & $\begin{array}{l}\text { Insulin } \\
\text { Resistance* }\end{array}$ & $\begin{array}{l}\text { Total } \\
\text { Cholesterol }^{+}\end{array}$ & Triglyceride $^{+}$ & LDL-c ${ }^{+}$ & HDL-c ${ }^{+}$ \\
\hline \multicolumn{6}{|l|}{ Protease Inhibitors } \\
\hline Amprenavir & $\leftrightarrow$ to $\uparrow$ & $\uparrow \uparrow$ & $\uparrow \uparrow$ & $\uparrow$ & $\uparrow$ \\
\hline Atazanavir & $\leftrightarrow$ & $\uparrow$ & $\leftrightarrow$ to $\uparrow$ & $\leftrightarrow$ to $\uparrow$ & $\leftrightarrow$ to $\uparrow$ \\
\hline Indinavir & $\uparrow \uparrow \uparrow$ & $\uparrow$ & $\leftrightarrow$ to $\uparrow$ & $\uparrow \uparrow$ & $\leftrightarrow$ \\
\hline Lopinavir/Ritonavir & $\leftrightarrow$ & $\uparrow$ to $\uparrow \uparrow$ & $\uparrow \uparrow$ & $\uparrow \uparrow$ & $\leftrightarrow$ \\
\hline Nelfinavir & $\leftrightarrow$ to $\uparrow \uparrow$ & $\uparrow \uparrow$ & $\leftrightarrow$ to $\uparrow \uparrow$ & $\uparrow \uparrow$ & $\leftrightarrow$ \\
\hline Ritonavir & $\leftrightarrow$ & $\uparrow \uparrow \uparrow$ & $\uparrow \uparrow \uparrow$ & $\uparrow \uparrow \uparrow$ & $\leftrightarrow$ \\
\hline \multicolumn{6}{|c|}{ Non-Nucleoside Reverse Transcriptase Inhibitor } \\
\hline Efavirenz & $\downarrow \downarrow$ (switch) ${ }^{\#}$ & $\uparrow$ & $\downarrow \downarrow$ (switch) $)^{\#}$ & ND & $\uparrow \uparrow$ \\
\hline Nevirapine & $\downarrow \downarrow$ (switch) ${ }^{\#}$ & $\uparrow$ & $\leftrightarrow$ & $\uparrow$ & $\uparrow \uparrow \uparrow$ \\
\hline
\end{tabular}

* Insulin Resistance: The arrows refer to relative changes, not to specific percentages. Due to the differences in percent changes between various methods (HOMA and clamp), specific percentages have not been used.

${ }^{+}$Lipids: $\quad \uparrow$ or $\downarrow<20 \%, \quad \uparrow \uparrow$ or $\downarrow \downarrow 20-40 \%, \quad \uparrow \uparrow \uparrow$ or $\downarrow \downarrow \downarrow>40 \%$

\# (switch) refers to information obtained primarily from PI to NNRTI switch studies

ND: No adequate data available

Table 2: Effect of PIs on Lipid and Glucose Metabolism in HIV negative patients

\begin{tabular}{|c|c|c|c|c|c|}
\hline Drug & $\begin{array}{l}\text { Insulin } \\
\text { Resistance }^{*}\end{array}$ & $\begin{array}{l}\text { Total } \\
\text { Cholesterol }\end{array}$ & Triglyceride $^{+}$ & LDL-c $^{+}$ & HDL-c ${ }^{+}$ \\
\hline \multicolumn{6}{|l|}{ Protease Inhibitors } \\
\hline Amprenavir & ND & $\leftrightarrow$ & $\leftrightarrow$ & ND & ND \\
\hline Atazanavir & $\leftrightarrow$ & $\leftrightarrow$ & $\leftrightarrow$ & $\leftrightarrow$ & $\leftrightarrow$ \\
\hline Indinavir & $\uparrow \uparrow$ & $\leftrightarrow$ & $\leftrightarrow$ & $\leftrightarrow$ & $\leftrightarrow$ \\
\hline Lopinavir/ritonavir & $\leftrightarrow$ to $\uparrow \uparrow$ & $\leftrightarrow$ & $\uparrow \uparrow$ & $\leftrightarrow$ & $\leftrightarrow$ \\
\hline Ritonavir & ND & $\leftrightarrow$ & $\uparrow \uparrow \uparrow$ & $\leftrightarrow$ & $\downarrow$ \\
\hline
\end{tabular}

and negative subjects ${ }^{[33,36,38,57,58]}$; whether this is due to the LPV or the boosting dose of RTV is unclear.

Studies on the effect of NFV on TG metabolism have been conflicting; two studies show no change in TG levels ${ }^{[30,34]}$, while other studies show an increase of $42-50 \%$ in fasting TG levels ${ }^{[359,60]}$ Likewise, studies of APV have been conflicting; Sadler et al. showed that 7 days of APV in HIV negative patients had no effect on $\mathrm{TG}^{[61]}$, while Dube et al. showed that 48 weeks of APV in HIV positive patients increased TG by $90 \%{ }^{[35]}$. One explanation for this discrepancy is the difference in duration of treatment. Both $\operatorname{IDV}^{[30,33,59,60,62]}$ and $\mathrm{ATZ}^{[337,59,60,63]}$ have little or no significant effect on TG levels in both HIV negative and HIV positive patients.

VLDL-c: The effect of PIs on VLDL metabolism has not been studied as extensively. Behrens and Mulligan have both shown that PIs, as a group, increase VLDL levels in HIV infected subjects ${ }^{[16,29]}$. Other studies of VLDL levels have been done primarily in HIV negative patients; RTV raises VLDL-c levels by $159 \%{ }^{[55]}$, while LPV/r raises VLDL-c by $33 \%{ }^{[36]}$. Again, it is unclear whether LPV itself may cause this effect or whether it is due to the RTV component. In HIV negative patients, IDV did not change VLDL levels after 4 weeks of treatment $^{[33]}$ and ATZ did not change VLDL levels after five days of treatment ${ }^{[37]}$.
LDL-c: As a group, PIs are also associated with elevated LDL-c levels ${ }^{[17,21,29,53]}$. In HIV infected patients, IDV, RTV, NFV, APV and LPV/r have been shown to increase LDL-c levels by 11$50 \%{ }^{[30,34,3557,60,62]}$. However, this increase is probably due to restoration of health, rather than an intrinsic effect of PIs on LDL metabolism as it does not occur in HIV negative patients given PI. Multiple studies of RTV, IDV, LPV/r, or ATZ administration for durations of 5 days to 4 weeks to HIV negative patients have shown no change in LDL-c levels ${ }^{[33,36,37,55]}$.

Apo B: The few studies that analyzed the change in apoB levels (that associated with non-HDL-c, including VLDL, IDL and LDL) with the use of PIs have consistently shown an increase in apoB levels with the use of IDV, NFV, LPV/r, or RTV ${ }^{[34,55,67,62]}$ as well as PIs as a group ${ }^{[16]}$. Stable isotope studies have shown that patients on PI based therapy have significantly higher rates of apoB synthesis as well as a decrease in the transfer of triglyceride between VLDL species ${ }^{[64]}$.

HDL-c: In a majority of studies, PIs do not significantly change HDL-c levels ${ }^{[5,17,21,29]}$; one study of pooled PIs found a decrease in HDL-c. Some studies of individual PIs (such as IDV, NFV, APV, LPV/r and ATZ) ${ }^{[34,35,38,6062,63]}$ have shown small increases in HDL$\mathrm{c}$ with treatment, but there are an equal number of 
studies in both HIV positive and HIV negative patients indicating no change in HDL-c levels ${ }^{[30,33,3637,57,65]}$. As a group, PIs do not cause a change in apo A-1 (the apolipoprotein associated with HDL-c) ${ }^{[16]}$. Although a few solitary studies of LPV/r and $\mathrm{NFV}^{[34,57]}$ concluded that apo A-1 does increase with these drugs, these studies need to be further verified.

Mechanism of action: Although the exact mechanism by which PIs induce hypertriglyceridemia is not clear, it is most likely multifactorial. One possible mechanism involves the inhibition of proteosomal degradation of apolipoprotein B by PIs. Liang et al. demonstrated that certain PIs (saquinavir and RTV) inhibit the intracellular proteosomes which degrade nascent apolipoprotein $\mathrm{B}$, thereby resulting in the increased production and secretion of $\mathrm{TG}$ rich particles ${ }^{[66]}$. Another proposed mechanism for increased VLDL production is PI induction of increased levels of SREBP-1 ${ }^{[67,68]}$, which stimulates lipid synthesis. Other proposed mechanisms for hyperlipidemia ${ }^{[44,69]}$ are not as well supported.

It is also important to note that the dyslipidemia associated with PIs likely has a genetic component. For example, there are multiple reports regarding the association of apolipoprotein $\mathrm{E}$ alleles and the hyperlipidemia caused by PIs; Lister et al. reported a case of latent dysbetalipoproteinemia which was precipitated by PIs ${ }^{[70]}$ and Behrens reported a significant association between the apoE4 allele and hyperlipidemia in patients on PIs ${ }^{[71]}$. Another study indicating the synergism between genetic predisposition and pharmacological agents, done by Fauvel et al., showed that $40 \%$ of the variability in HDL and TG in HIV patients on PIs was explained by polymorphisms in the apo C-III gene ${ }^{[72]}$.

Effect of NNRTIs on lipid metabolism: The effect of EFV and NVP on lipid metabolism has been assessed in switch studies as well as a few prospective studies. It is clear that NNRTIs increase HDL by $15-49 \%^{[34,62,73,74]}$ and they also increase apo A-1 levels ${ }^{[34,62]}$. The effect of NNRTIs on other lipids has been more variable. In prospective studies, NVP causes an increase in LDL (likely due to restoration to health) ${ }^{[62]}$; in switch studies where patients are switched from PI based treatment to NVP based treatment, LDL levels have been unchanged $^{[51,74]}$. NVP does not change TG levels in prospective studies ${ }^{[34,62]}$ and decreases TG levels in switch studies ${ }^{[49,73-75]}$. The effect of EFV on LDL-c and TG levels has been variable due to differences in measurement techniques (fasting vs. non-fasting lipid values) and lack of statistical analysis; it is possible that EFV improves TG in switch studies ${ }^{[50]}$.

\section{CONCLUSION}

Abnormalities in glucose and lipid metabolism are not uncommon in HIV patients who are on HAART. Some changes, such as increased triglyceride levels and decreased HDL-c and LDL-c levels, are seen with HIV infection alone. The use of HAART and specifically, the use of PIs, has been associated with insulin resistance, hypertriglyceridemia and elevated LDL levels in some patients. It is likely that these metabolic effects are due to a combination of genetic predisposition in patients, restoration of health and the effects of individual drugs. The changes in glucose metabolism are predominantly a reflection of the pharmacological blockade of the GLUT-4 glucose transporter, but other mechanisms (such as increased hepatic glucose production and decreased insulin secretion) may also play a role. The increase in $\mathrm{TG}$ levels is related to specific drugs. The increase in LDL$\mathrm{c}$ is likely due to restoration of health, as it occurs with both PI and NNRTI-based HAART in HIV-infected patients, but not in HIV-negative subjects given PI. HDL levels do not change significantly with PI-based HAART, but increase with NNRTI. With respect to the individual medications, IDV causes the most insulin resistance, while ATZ likely causes the least. RTV is most associated with hypertriglyceridemia, while IDV and ATZ are the least associated. Although further studies are required in order to elucidate mechanisms, diabetes and hyperlipidemia in this population of patients should be aggressively treated to decrease the risk of cardiovascular disease.

\section{REFERENCES}

1. Palella, F.J.Jr., K.M. Delaney and A.C. Moorman et al., 1998. Declining morbidity and mortality among patients with advanced human immunodeficiency virus infection. HIV Outpatient Study Investigators. N. Engl. J. Med., 338: 853-60.

2. Dube, M.P., D.L. Johnson, J.S. Currier and J.M. Leedom, 1997. Protease inhibitor-associated hyperglycaemia. Lancet, 350: 713-4.

3. Sullivan, A.K. and M.R. Nelson, 1997. Marked hyperlipidaemia on ritonavir therapy. AIDS, 11: 938-9.

4. Lo, J.C., K. Mulligan, V.W. Tai, H. Algren and M. Schambelan, 1998. Buffalo hump in men with HIV-1 infection. Lancet, 351: 867-70.

5. Carr, A., K. Samaras and S. Burton et al., 1998. A syndrome of peripheral lipodystrophy, hyperlipidaemia and insulin resistance in patients receiving HIV protease inhibitors. AIDS, 12: F51-8.

6. Hommes, M.J., J.A. Romijn, E. Endert, J.K. Eeftinck Schattenkerk and H.P. Sauerwein, 1991. Insulin sensitivity and insulin clearance in human immunodeficiency virus-infected men. Metab. Clin. Exp., 40: 651-6.

7. Mulligan, K., C. Grunfeld, M.K. Hellerstein, R.A. Neese and M. Schambelan, 1993. Anabolic effects of recombinant human growth hormone in patients with wasting associated with human immunodeficiency virus infection. J. Clin. Endocrinol. Metab., 77: 956-62. 
8. Heyligenberg, R., J.A. Romijn, M.J. Hommes, E. Endert, J.K. Eeftinck Schattenkerk and H.P. Sauerwein, 1993. Non-insulin-mediated glucose uptake in human immunodeficiency virus-infected men. Clin. Sci., 84: 209-16.

9. Grunfeld, C., M. Pang, W. Doerrler, J.K. Shigenaga, P. Jensen and K.R. Feingold, 1992. Lipids, lipoproteins, triglyceride clearance and cytokines in human immunodeficiency virus infection and the acquired immunodeficiency syndrome. J. Clin. Endocrinol. Metab., 74: 104552.

10. Shor-Posner, G., A. Basit and Y. Lu et al., 1993. Hypocholesterolemia is associated with immune dysfunction in early human immunodeficiency virus-1 infection [see comments]. Am. J. Med., 94: 515-9.

11. Zangerle, R., M. Sarcletti, H. Gallati, G. Reibnegger, H. Wachter and D. Fuchs, 1994. Decreased plasma concentrations of HDL cholesterol in HIV-infected individuals are associated with immune activation. J. Acquir. Immune. Defic. Syndr., 7: 1149-56.

12. Fernandez-Miranda, C., F. Pulido and J.L. Carrillo et al., 1998. Lipoprotein alterations in patients with HIV infection: relation with cellular and humoral immune markers. Clin. Chim. Acta, 274: 63-70.

13. Grunfeld, C., D.P. Kotler, R. Hamadeh, A. Tierney, J. Wang and R.N. Pierson, 1989. Hypertriglyceridemia in the acquired immunodeficiency syndrome. Am. J. Med., 86: 27-31.

14. Hellerstein, M.K., C. Grunfeld and K. Wu et al., 1993. Increased de novo hepatic lipogenesis in human immunodeficiency virus infection. J. Clin. Endocrinol. Metab., 76: 559-65.

15. Feingold, K., R. Krauss, M. Pang, W. Doerrler, P. Jensen and C. Grunfeld, 1993. The hypertriglyceridemia of acquired immunodeficiency syndrome is associated with an increased prevalence of low density lipoprotein subclass pattern B. J. Clin. Endocrinol. Metab., 76: 1423-1427.

16. Behrens, G., A. Dejam and H. Schmidt et al., 1999. Impaired glucose tolerance, beta cell function and lipid metabolism in HIV patients under treatment with protease inhibitors. AIDS, 13: F63-70.

17. Woerle, H.J., P.R. Mariuz and C. Meyer et al., 2003. Mechanisms for the deterioration in glucose tolerance associated with HIV protease inhibitor regimens. Diabetes, 52: 918-25.

18. Beatty, G., M. Khalili and F. Abbasi et al., 2003. Quantification of insulin-mediated glucose disposal in HIV-infected individuals: comparison of patients treated and untreated with protease inhibitors. J. Acquir. Immune. Defic. Syndr., 33: 34-40.
19. Steil, G.M., J. Murray, R.N. Bergman and T.A. Buchanan, 1994. Repeatability of insulin sensitivity and glucose effectiveness from the minimal model. Implications for study design. Diabetes, 43: 1365-71.

20. Matthews, D.R., J.P. Hosker, A.S. Rudenski, B.A. Naylor, D.F. Treacher and R.C. Turner, 1985. Homeostasis model assessment: insulin resistance and beta-cell function from fasting plasma glucose and insulin concentrations in man. Diabetologia, 28: 412-9.

21. Visnegarwala, F., K.L. Krause and D.M. Musher, 1997. Severe diabetes associated with protease inhibitor therapy. Ann. Intern. Med., 127: 947.

22. Walli, R., O. Herfort and G.M. Michl et al., 1998. Treatment with protease inhibitors associated with peripheral insulin resistance and impaired oral glucose tolerance in HIV-1-infected patients. AIDS, 12: F167-73.

23. Moyle, G.J. and C. Baldwin, 1999. Lipid abnormalities during saquinavir soft-gel-based highly active antiretroviral therapy [letter]. J. Acquir. Immune. Defic. Syndr., 21: 423-4.

24. Tsiodras, S., C. Mantzoros, S. Hammer and M. Samore, 2000. Effects of protease inhibitors on hyperglycemia, hyperlipidemia and lipodystrophy: A 5-year cohort study. Arch. Intern. Med., 160: 2050-6.

25. Carr, A., K. Samaras, A. Thorisdottir, G.R. Kaufmann, D.J. Chisholm and D.A. Cooper, 1999. Diagnosis, prediction and natural course of HIV-1 protease-inhibitor-associated lipodystrophy, hyperlipidaemia and diabetes mellitus: a cohort study. Lancet, 353: 2093-9.

26. Dever, L.L., P.A. Oruwari, W.E. Figueroa, C.A. O'Donovan and R.H. Eng, 2000. Hyperglycemia associated with protease inhibitors in an urban HIV-infected minority patient population. Ann. Pharmacother., 34: 580-4.

27. Saves, M., F. Raffi and J. Capeau et al., 2002. Factors related to lipodystrophy and metabolic alterations in patients with human immunodeficiency virus infection receiving highly active antiretroviral therapy. Clin. Infect. Dis., 34: 1396-405.

28. Dube, M.P., H. Edmondson-Melancon, D. Qian, R. Aqeel, D. Johnson and T.A. Buchanan, 2001. Prospective evaluation of the effect of initiating indinavir-based therapy on insulin sensitivity and b-cell function in HIV-infected patients. J. Acquir. Immune. Defic. Syndr., 27: 130-4.

29. Mulligan, K., C. Grunfeld and V.W. Tai et al., 2000. Hyperlipidemia and insulin resistance are induced by protease inhibitors independent of changes in body composition in patients with HIV infection. J. Acquir. Immune. Defic. Syndr., 23: 35-43. 
30. Periard, D., A. Telenti and P. Sudre et al., 1999. Atherogenic dyslipidemia in HIV-infected individuals treated with protease inhibitors. The Swiss HIV Cohort Study. Circulation, 100: 700-5.

31. Bongiovanni, M., T. Bini, E. Chiesa, P. Cicconi, F. Adorni and A. Monforte d'Arminio, 2004. Lopinavir/ritonavir vs. indinavir/ritonavir in antiretroviral naive HIV-infected patients: immunovirological outcome and side effects. Antiviral Res., 62: 53-6.

32. Noor, M.A., T. Seneviratne and F.T. Aweeka et al., 2002. Indinavir acutely inhibits insulin-stimulated glucose disposal in humans: a randomized, placebo-controlled study. AIDS, 16: F1-8.

33. Noor, M.A., J.C. Lo and K. Mulligan et al., 2001. Metabolic effects of indinavir in healthy HIVseronegative men. AIDS, 15: F11-F18.

34. Fisac, C., N. Virgili and E. Ferrer et al., 2003. A comparison of the effects of nevirapine and nelfinavir on metabolism and body habitus in antiretroviral-naive human immunodeficiency virus-infected patients: A randomized controlled study. J. Clin. Endocrinol. Metab., 88: 5186-92.

35. Dube, M.P., D. Qian and H. Edmondson-Melancon et al., 2002. Prospective, intensive study of metabolic changes associated with 48 weeks of amprenavir-based antiretroviral therapy. Clin. Infect. Dis., 35: 475-81.

36. Lee, G.A., T. Seneviratne and M.A. Noor et al., 2004. The metabolic effects of lopinavir/ritonavir in HIV-negative men. AIDS, 18: 641-649.

37. Noor, M.A., R.A. Parker and E. O'Mara et al., 2004. The Effects of HIV Protease Inhibitors Atazanavir and Lopinavir/ritonavir on Insulin Sensitivity in HIV-seronegative Healthy Adults. AIDS, (in press).

38. Martinez, E., P. Domingo and M.J. Galindo et al., 2004. Risk of metabolic abnormalities in patients infected with HIV receiving antiretroviral therapy that contains lopinavir-ritonavir. Clin. Infect. Dis., 38:1017-23..

39. Voigt, E., J.C. Wasmuth and M. Vogel et al., 2004. Safety, efficacy and development of resistance under the new protease inhibitor lopinavir/ritonavir: 48-week results. Infection, 32: 82-8.

40. Lee, G.A., D.D. Mafong and M.A. Noor et al., 2004. HIV protease inhibitors increase adiponectin levels in HIV-negative men. J. Acquir. Immune Defic. Syndr., 36: 645-647.

41. Murata, H., P.W. Hruz and M. Mueckler, 2000. The mechanism of insulin resistance caused by HIV protease inhibitor therapy. J. Biol. Chem., 275: 20251-20254.

42. Nolte, L.A., K.E. Yarasheski, K. Kawanaka, J. Fisher, N. Le and J.O. Holloszy, 2001. The Hiv protease inhibitor indinavir decreases insulin- and contraction-stimulated glucose transport in skeletal muscle. Diabetes, 50: 1397-401.
43. Rudich, A., S. Vanounou and K. Riesenberg et al., 2001. The HIV protease inhibitor nelfinavir induces insulin resistance and increases basal lipolysis in 3T3-L1 adipocytes. Diabetes, 50: 142531.

44. Ranganathan, S. and P.A. Kern, 2002. The HIV protease inhibitor saquinavir impairs lipid metabolism and glucose transport in cultured adipocytes. J. Endocrinol., 172: 155-62.

45. Murata, H., P.W. Hruz and M. Mueckler, 2002. Indinavir inhibits the glucose transporter isoform Glut4 at physiologic concentrations. AIDS, (in press).

46. Koster, J.C., M.S. Remedi, H. Qiu, C.G. Nichols and P.W. Hruz, 2003. HIV protease inhibitors acutely impair glucose-stimulated insulin release. Diabetes, 52: 1695-700.

47. Dufer, M., Y. Neye, P. Krippeit-Drews and G. Drews, 2004. Direct interference of HIV protease inhibitors with pancreatic beta-cell function. Naunyn Schmiedebergs Arch. Pharmacol., 369: 583-90.

48. Schwarz, J.M., G.A. Lee and S. Park et al., 2004. Indinavir increases glucose production in healthy HIV-negative men. AIDS, (in press).

49. Martinez, E., I. Conget, L. Lozano, R. Casamitjana and J.M. Gatell, 1999. Reversion of metabolic abnormalities after switching from HIV-1 protease inhibitors to nevirapine. AIDS, 13: 805-10.

50. Martinez, E., M.A. Garcia-Viejo and J.L. Blanco et al., 2000. Impact of switching from human immunodeficiency virus type 1 protease inhibitors to efavirenz in successfully treated adults with lipodystrophy. Clin. Infect. Dis., 31: 1266-73.

51. Domingo, P., X. Matias-Guiu and R.M. Pujol et al., 2001. Switching to nevirapine decreases insulin levels but does not improve subcutaneous adipocyte apoptosis in patients with highly active antiretroviral therapy-associated lipodystrophy. J. Infect. Dis., 184: 1197-201.

52. Calza, L., R. Manfredi, B. Farneti and F. Chiodo, 2003. Incidence of hyperlipidaemia in a cohort of 212 HIV-infected patients receiving a protease inhibitor-based antiretroviral therapy. Intl. J. Antimicrob. Agents, 22: 54-9.

53. Walli, K., G. Michl and S. Segerer, 1999. Dyslipidemia and insulin resistance in HIVinfected patients treatd with reverse transcriptase inhibitors alone and in combination with protease inhibitors. 6th Conf. Retroviruses and Opportunistic Infections. Chicago, IL.

54. Danner, S.A., A. Carr and J.M. Leonard et al., 1995. A short-term study of the safety, pharmacokinetics and efficacy of ritonavir, an inhibitor of HIV-1 protease. European-Australian Collaborative Ritonavir Study Group. N. Engl. J. Med., 333: 1528-33. 
55. Purnell, J.Q., A. Zambon and R.H. Knopp et al., 2000. Effect of ritonavir on lipids and post-heparin lipase activities in normal subjects. AIDS, 14: 51-7.

56. Cameron, D.W., M. Heath-Chiozzi and S. Danner et al., 1997. Randomised placebo-controlled trial of ritonavir in advanced HIV-1 disease. The Advanced HIV Disease Ritonavir Study Group [see comments]. Lancet, 351: 543-9.

57. Badiou, S., C. Merle De Boever, A.M. Dupuy, V. Baillat, J.P. Cristol and J. Reynes, 2003. Decrease in LDL size in HIV-positive adults before and after lopinavir/ritonavir-containing regimen: an index of atherogenicity? Atherosclerosis, 168: 107-13.

58. Gonzalez de Requena, D., F. Blanco, T. GarciaBenayas, I. Jimenez-Nacher, J. Gonzalez-Lahoz and V. Soriano, 2003. Correlation between lopinavir plasma levels and lipid abnormalities in patients taking lopinavir/ritonavir. AIDS Patient Care STDS, 17: 443-5.

59. Sanne, I., P. Piliero, K. Squires, A. Thiry and S. Schnittman, 2003. Results of a phase 2 clinical trial at 48 weeks (AI424-007): a dose-ranging, safety and efficacy comparative trial of atazanavir at three doses in combination with didanosine and stavudine in antiretroviral-naive subjects. J. Acquir. Immune. Defic. Syndr., 32: 18-29.

60. Murphy, R.L., I. Sanne and P. Cahn et al., 2003. Dose-ranging, randomized, clinical trial of atazanavir with lamivudine and stavudine in antiretroviral-naive subjects: 48-week results. AIDS, 17: 2603-14.

61. Sadler, B.M., P.J. Piliero, S.L. Preston, P.P. Lloyd, Y. Lou and D.S. Stein, 2001. Pharmacokinetics and safety of amprenavir and ritonavir following multiple-dose, co-administration to healthy volunteers. AIDS, 15: 1009-18.

62. van der Valk, M., J.J. Kastelein and R.L. Murphy et al., 2001. Nevirapine-containing antiretroviral therapy in HIV-1 infected patients results in an anti-atherogenic lipid profile. AIDS, 15: 2407-14.

63. Squires, K., A. Lazzarin and J.M. Gatell et al., 2004. Comparison of once-daily atazanavir with efavirenz, each in combination with fixed-dose zidovudine and lamivudine, as initial therapy for patients infected with HIV. J. Acquir. Immune. Defic. Syndr., 36: 1011-1019.

64. Schmitz, M., G.M. Michl and R. Walli et al., 2001. Alterations of apolipoprotein B metabolism in $\mathrm{HIV}$-infected patients with antiretroviral combination therapy. J. Acquir. Immune. Defic. Syndr., 26: 225-35.

65. Roberts, A.D., R.A. Muesing, D.M. Parenti, J. Hsia, A.G. Wasserman and G.L. Simon, 1999. Alterations in serum levels of lipids and lipoproteins with indinavir therapy for human immunodeficiency virus-infected patients. Clin. Infect. Dis., 29: 441-3.
66. Liang, J.S., O. Distler and D.A. Cooper et al., 2001. HIV protease inhibitors protect apolipoprotein B from degradation by the proteasome: a potential mechanism for protease inhibitor-induced hyperlipidemia. Nat. Med., 7: 1327-31.

67. Riddle, T.M., D.G. Kuhel, L.A. Woollett, C.J. Fichtenbaum and D.Y. Hui, 2001. HIV protease inhibitor induces fatty acid and sterol biosynthesis in liver and adipose tissues due to the accumulation of activated sterol regulatory element-binding proteins in the nucleus. J. Biol. Chem., 276: 375149.

68. Bastard, J.P., M. Caron and H. Vidal et al., 2002. Association between altered expression of adipogenic factor SREBP1 in lipoatrophic adipose tissue from HIV-1-infected patients and abnormal adipocyte differentiation and insulin resistance. Lancet, 359: 1026-31.

69. Carr, A., K. Samaras, D.J. Chisholm and D.A. Cooper, 1998. Pathogenesis of HIV-1-protease inhibitor-associated peripheral lipodystrophy, hyperlipidaemia and insulin resistance. Lancet, 351: 1881-3.

70. Lister, R.K., M. Youle, D.R. Nair, A.F. Winder and M.H. Rustin, 1999. Latent dysbetalipoproteinaemia precipitated by HIV-protease inhibitors. Lancet, 353: 1678.

71. Behrens, G., H.H. Schmidt, M. Stoll and R.E. Schmidt, 1999. ApoE genotype and proteaseinhibitor-associated hyperlipidaemia. Lancet, 354: 76.

72. Fauvel, J., E. Bonnet and J.B. Ruidavets et al., 2001. An interaction between apo C-III variants and protease inhibitors contributes to high triglyceride/low HDL levels in treated HIV patients. AIDS, 15: 2397-406.

73. Negredo, E., J. Ribalta and R. Paredes et al., 2002. Reversal of atherogenic lipoprotein profile in HIV1 infected patients with lipodystrophy after replacing protease inhibitors by nevirapine. AIDS, 16: 1383-9.

74. Tebas, P., K. Yarasheski and K. Henry et al., 2004. Evaluation of the virological and metabolic effects of switching protease inhibitor combination antiretroviral therapy to nevirapine-based therapy for the treatment of HIV infection. AIDS Res. Hum. Retroviruses, 20: 589-94.

75. Ruiz, L., E. Negredo and P. Domingo et al., 2001. Antiretroviral treatment simplification with nevirapine in protease inhibitor-experienced patients with hiv-associated lipodystrophy: 1-year prospective follow-up of a multicenter, randomized, controlled study. J. Acquir. Immune. Defic. Syndr., 27: 229-36. 